\title{
La complejidad en la educación del siglo XXI
}

\author{
Complexity in 21st Century Education
}

\author{
Ofelia Berrido \\ Directora de la Unidad de Evaluación y Desarrollo Docente, \\ y del Departamento de Tecnología Educativa UNPHU \\ Santo Domingo, República Dominicana \\ oberrido@unphu.edu.do
}

Fecha de recepción: 21 de junio de 2017

Fecha de aceptación: 20 de julio de 2017

Favor de citar este artículo de la siguiente forma:

Berrido, O. (2017). La complejidad en la educación del siglo XXI.

Revista AULA. Vol. 61, Número 1, julio-diciembre 2017. Santo Domingo: Amigo del Hogar

\section{RESUMEN}

En este ensayo se reflexiona sobre el impacto del paradigma de la complejidad en el mundo de la educación del siglo XXI busca demostrar el cómo la educación, para ser efectiva y eficiente, debe contemplar las acciones que ejecutará sobre las variables críticas que impactan el sistema educacional. En el trabajo se han tocado los siguientes puntos críticos: El gobierno como líder del sistema y como ente definitorio de las necesidades nacionales; las universidades como responsables de la implementación certera de la función social de formar a los ciudadanos y de ofertar una educación con un estilo propio dentro de la autonomía que el sistema del país permite en busca de una educación libre, no dictatorial; los profesores como parte vital del sistema por ser guías o facilitadores que dirigen el proceso en el lugar donde se hace efectivo el sistema completo, en las aulas tanto presenciales como virtuales; y finalmente, los estudiantes, alfa y omega para quien se inicia y termina el proceso en busca de profesionales que hagan de la nación un lugar productivo en el que se vive una relación basada en valores éticos. Para ello reflexionamos tomando como marco de referencia investigaciones previas sobre el tema y culturas milenarias que aún se expresan soterradamente en el accionar del ser humano de este siglo.

Palabras Clave: Educación, Competencias, Complejidad, Efectividad, Eficiencia, Siglo XXI

\begin{abstract}
This essay reflects on the impact of the paradigm of complexity in the world of the XXI century's education to demonstrate how education, to be effective and efficient, must contemplate the actions that it will execute on the critical variables that impact its system. The paper has touched the following critical points: Government as the leader of the system and as a defining entity of national needs; universities as responsible for the accurate implementation of the social function of educating citizens, as well as, for offering an education with its own style, within the autonomy that the country system allows, as representatives of particular visions in search of a free, non dictatorial education; teachers as a vital part of the system because they are the guides or facilitators who direct the process where the complete system is implemented, in both classrooms and virtual classes. And finally, the students, for whom the process exist in search of professionals who make of the nation a productive place to live and work based on ethical values. Our reflections uses as a framework of references previous researches on the subject and the wisdom of millennial cultures that still in the present century expresses in the hearts and actions of today mankind.
\end{abstract}

Keywords: Education of the 21st Century, Complexity, Competences, Effectiveness, Efficiency 


\section{La complejidad en la educación del siglo XXI \\ Complexity in 21st Century Education}

Estamos inmersos en el periodo de plena efervescencia de la revolución científica y tecnológica del siglo XXI. Este tipo de transformación se caracteriza por ser fuente de cambios de los paradigmas previamente aceptados. Vivimos una nueva manera de pensar, abordar, producir y hasta de asimilar distintas realidades. Las necesidades, las situaciones que la época plantea y los problemas teórico-prácticos que surgen demandan cambios y rupturas con las viejas formas de ver y enfrentar el mundo. Nuestra antigua racionalidad occidental se ha vuelto rancia. Y cuando queremos penetrar las raíces mismas del raciocinio tradicional y procedemos a hacernos las siguientes preguntas en busca de respuestas que apliquen a las necesidades de este siglo: ¿Quién razona?, ¿sobre qué y con qué instrumentos? Y sobre todo, ¿para qué razona? Entonces, terminamos recordando una de las frases del filósofo alemán Friedrich Schlegel (como se cita en Suzuki, 1991): "La racionalidad tradicional se encierra a sí misma hasta el auto aniquilamiento, llevada a su extremo, eliminando el pensamiento de justificación desde las ideas mismas".

Se puede puntualizar la afirmación anterior con ejemplos provenientes de distintas disciplinas que han probado la realidad de la interdependencia. Entre ellos, conceptos que ya desde hace unos 2,000 años eran conocidos en las profundidades de las montañas del Tíbet: "Como es arriba es abajo y como es afuera es adentro". Son maneras en que Oriente explica el mundo. Ya los grandes maestros Zen conocían y explicaban el concepto del holismo cuando afirmaban que el todo está contenido en sus partes y las partes en el todo. De igual forma, desde los primeros siglos Oriente entiende el vacío como el centro de la verdad, el lugar de origen y el fin del todo y de la nada.

Nagarjuna en el siglo II manifestó que el vacío es el fundamento de todo, porque gracias a este, todo es posible. Sostuvo que las cosas carecen de naturaleza propia y son por tanto vacías, estableciendo una equivalencia entre la falta de naturaleza propia y la vacuidad de las cosas (citado por Arnaud, 2011). Hoy, la ciencia de Occidente lo acepta y lo plantea como un descubrimiento propio. Edgar Morin (2003, p. 341-342), respetado investigador y filósofo lo reclasifica alrededor de 1999 con el nombre del "Paradigma Perdido" y entonces hablamos del pensamiento complejo y de los sistemas complejos e interconectados".

No hay separación entre lo absoluto y lo relativo, entre la realidad y las apariencias, entre la fuente de origen y el universo manifiesto. Nada es real, en el sentido en que nada tiene una existencia independiente que le pertenezca como propia, y no es más que un fenómeno transitorio, combinación de millones de fenómenos todavía más fugaces. "La forma es solo vacío, el vacío solo forma": vacio en tanto no tiene existencia propia, todo es interdependiente. Estas palabras contenidas en el Sutra del Corazón hoy se convierten en la Teoría de Sistemas. El concepto es oriental y Occidente no lo había entendido hasta que filósofos como el ya mencionado Edgar Morín (2003), pensador francés de origen judío sefardí le da vida y 
mientras para Occidente se constituye en un nuevo paradigma; para Oriente es parte de su cultura milenaria.

Quizás sea de utilidad para el entendimiento de lo planteado el penetrar un poco en el concepto de vacío e interdependencia. Cuando hablamos de que todo efecto tiene su causa y que toda causa tiene su efecto (ley de causa y efecto) podemos acercarnos al hecho de que nada tiene existencia propia porque todo proviene de algo. El objeto no existiría sin la causa que le dio origen, entonces él por sí mismo no tiene existencia propia: está vacío de existencia propia. Es interdependiente porque depende de una o múltiples causas. Todos los objetos están interrelacionados con los objetos de los cuales dependen: son interdependientes. Las cosas no son tan simples como las vemos sino que hay una complejidad oculta.

Oriente y Occidente han tenido una larga historia de ver las cosas de forma diferente y desde un punto de vista en ocasiones, totalmente opuestos. De hecho, y como manifestación de lo antes aseverado hasta la naturaleza se confabula: por el Este se eleva el sol y por el Oeste se pone; en Occidente Dios se encuentra afuera en el cielo; mientras, en Oriente Dios se encuentra dentro del ser; el luto es negro, mientras que en Oriente es blanco; nuestra medicina es química mientras la suya es energética; nuestra cultura es competitiva mientras que la de ellos es grupal y cooperativa.

Centrémonos en "el vacío" y la forma en que estas culturas afrontan el concepto. Cuando hablamos del vacío en el sentido ordinario del término hablamos de la ausencia total de materia. Si bien en el siglo XVII se creía que el vacío no formaba parte de la naturaleza, hoy la ciencia occidental asume el vacío a tal punto que se acepta que este prima sobre la materia.
Y así vamos viendo cómo el conocimiento se hace cada vez más complejo. La complejidad es una nueva epistemología que se escapa de la tradicional dualidad cartesiana del alma y el cuerpo: donde el alma (mente) es capaz de discernir entre lo bueno y lo malo y donde el cuerpo se reduce a leyes mecánicas. Y es que el hombre no es un ente separado y dual sino que forma parte del todo que lo impacta y que a la vez él impacta. Es parte de los sistemas que forman el todo, y a la vez, impacta el todo. El bien y el mal no están separados del ser sino que son parte del todo del cual él forma parte.

La noción de separatividad cartesiana limita el conocimiento porque separa el todo en partes para su conocimiento. Descartes buscaba descomponer para conocer los elementos en su simplicidad, pero hoy las ciencias sistémicas religan lo que está separado en busca de entender el todo que se autoorganiza y autoproduce constantemente. Otro aspecto de la separatividad es el de la división entre el objeto observado y el observador. Hoy, se sabe que el observador no solo interfiere sino que puede afectar o alterar lo observado. Morin, por su parte, afirma que el pensamiento complejo apela a la dialógica que utiliza lo separable, pero lo inserta en lo inseparable (2010, p.132).

Los seres y las cosas provienen de sistemas que se entrelazan y que ocultan en sus partes las verdades del todo, no observables a simple vista. No existe la dualidad. Cuando observamos un átomo su electrón no tiene existencia propia como no lo tiene el neutrón o el positrón (antimateria) son simplemente partes del todo, en este caso del átomo. Pero sigamos con el tema a modo de un juego lúdico: En todo bien hay un mal oculto, en todo mal existe el bien como lo muestra el símbolo del Taoismo. Pero, ¿Qué es el alma y el cuerpo sino parte de un todo? El cuerpo no existe sin 
esa parte almática (mente). ¿A quién soporta el alma sino al cuerpo?, son interdependientes, no existe uno sin el otro. Pero el cuerpo físico tampoco tiene dependencia propia; surge de la unión de células masculinas y femeninas que en una interdependencia sin fin, forman la humanidad. Todo lo existente es interdependiente. Como podemos ver, los docentes y los estudiantes están enfrentados a nuevas formas de ver el mundo. La mirada de hoy no se queda en la superficie porque la ciencia tradicional de dividir el todo en sus partes para estudiarlas ya no nos da suficientes respuestas. Hay verdades ocultas en "las partes" que el ser humano con el pensamiento lógico y lineal no ha podido develar.

Entonces, se necesita una mirada diferente que vea a través del tiempo (diacronía) y que pueda observar y analizar los múltiples hechos que suceden en el mismo tiempo (sincronía) que les ha tocado vivir para con un ojo analítico crítico ver más allá de las apariencias. Es ahí donde la educación de hoy debe poner sus ojos en busca de un aprendizaje orientado a desarrollar el arte y la ciencia del descubrimiento y de la invención. Y así, resolver problemas perentorios de la sociedad y el mundo, mediante la creatividad y el pensamiento lateral o pensamiento divergente en busca de generar ideas mediante la exploración de un sinnúmero de posibles soluciones. Este debe ser el fin de toda enseñanza moderna porque estos sistemas no lineales contienen realidades ocultas que están esperando ser develadas y son un verdadero reto para la educación y el mundo de la investigación.

Es así como la información y el conocimiento cambian a través de las edades: desde los nuevos significados del tiempo y espacio; la física cuántica; las nuevas perspectivas sobre la incertidumbre y la nueva relación entre el sujeto y objeto del conocimiento; las investigaciones de H. Maturana sobre la autopoiesis del ser (reproducción y auto regeneración); la interrelación entre cultura y medioambiente; la "teoría general de sistemas" que hoy resurge como novedad; la nueva manera de abordar las matemáticas, la "teoría del caos", y la de los fractales, las nuevas dimensiones, la robótica, la nanotecnología y en fin... El siglo XXI interpela las nuevas realidades con una forma diferente de pensar y vivir el mundo donde las partes y sistemas son interdependientes y complementarios.

Este mundo de la complejidad en todos los órdenes es el panorama que se le presenta a los nuevos docentes y estudiantes del siglo XXI. $Y$ en el mundo maravilloso de World Wide Web todo está ahí para ser visto, analizado, entendido y aplicado para la toma de decisiones.

La cantidad de información y conocimiento que se nos presenta es descomunal. Por ende, se hace imprescindible una nueva manera de abordar todo esto en el universo de la Educación porque no existe posibilidad alguna de que la finita mente humana domine todo el conocimiento de la humanidad. ¡Demasiada información para ser digerida en la pequeña unidad de tiempo de una vida! Entonces, hay que modificar las viejas formas y estructuras de enseñanza y aprendizaje de la Educación Superior o crear nuevas que faciliten el proceso $\mathrm{y}$ aseguren su pertinencia y calidad.

El proceso de disrupción entre los viejos y nuevos paradigmas lo cambia todo. Justamente por ello, son los gobiernos con sus encuestas y censos nacionales, con sus estudios económicos y sociales, con las demandas provenientes de las necesidades nacionales y globales y sus planes estratégicos quienes deben decidir qué es, de toda aquella información y del extenso conocimiento de la humanidad, lo que se debe 
incluir en los currículos nacionales. ¿Cuál es el perfil general del profesional que necesita el país?; ¿cuáles son los currículos por carrera?; ¿cuáles los perfiles por carreras que el gobierno con sus expertos en cada ramo define y puede entonces entregar para que las universidades puedan trabajar los planes de estudios enfocados en las necesidades nacionales, y que a su vez, puedan asegurar un profesional capaz de ejercer en cualquier parte del mundo? Y sobre todo, ¿qué papel juega la ética y el bien común en esos planes?

Las universidades, en base al currículo que entrega el gobierno, procederán a crear los planes de estudios de carreras y maestrías que incluyen los programas y sílabus correspondientes y le darán a todo aquello el talante que representa la institución en base a los planes estratégicos de la universidad, su misión, visión y los principios y valores que la rigen. Todo ello, bajo los lineamientos de un modelo educativo basado en competencias que incluya, además de lo puramente académico, el desarrollo de un ser humano íntegro, productivo, preocupado por la sociedad en que vive y la naturaleza que sirve de sustento a la vida.

Hoy por hoy, el Ministerio de Educación Superior está concentrado en asegurar la calidad y la eficacia de la enseñanza con una producción de egresados que supla las exigencias sociales y económicas de la República Dominicana, y que le permita a los jóvenes de hoy volver realidad sus sueños de convertirse en entes productivos que aporten al bienestar espiritual y material de sus familias y la sociedad que los alberga.

Los retos son muchos porque el resultado final depende de múltiples variables que convierten el flujo del proceso en un camino lleno de complejidades. Y es que la calidad de la educación se ve impactada, según los estudios realizados por la Organización para la Cooperación y el Desarrollo Económico (2008, citado en MEPYD, 2010), por la falta de preparación de los estudiantes de nuevo ingreso; las altas tasas de abandono con una media desde el comienzo hasta la conclusión de una carrera de un 50\% y por el hecho de que, según la misma fuente, la participación en educación superior corresponde al bajo número de un $29.1 \%$ de los estudiantes entre $18-24$ años de edad. Como podemos ver, el asunto es, en verdad, complejo.

En cuanto a la calidad docente, juegan un papel importante los criterios de selección y contratación de los profesores; la educación continua; los programas de certificación; el acceso a becas; un salario que le permita ejercer su carrera sin preocupaciones de otro orden; la contratación de profesores expertos $\mathrm{y}$ activos en el mundo laboral de su rama para que hablen en las aulas desde el seno de la experiencia. Todo esto permite que trabajen en una sola institución educativa con tiempo disponible para estudiar, escribir y gestionar de manera adecuada el proceso de enseñanza y aprendizaje y, al mismo tiempo, compartir con su familia en busca de la armonía que como ser humano le permita mejorar su desempeño.

Estudios internacionales realizados por la OECD (2014, p. 198), en los cuales no fue incluida República Dominicana, revelan que el salario de los profesores representa el costo de inversión más alto en la educación formal, pero hay un detalle importante que debemos tomar en cuenta pues nos parece que puede afectar directamente la calidad de la educación: según las estadísticas presentadas en dicho informe, el salario es directamente proporcional a lo atractivo de la profesión e influye por igual en la deserción de profesores. Así, podemos deducir que a menor salario, probablemente, los candidatos no sean los de mayor preparación; 
del mismo modo, los de mayor preparación, posiblemente, serían los que se retiren temprano en busca de otras fuentes de sustento económico quedando afectada la calidad de la educación.

En otro orden de ideas, los empresarios se quejan, aluden que no reciben en sus empresas egresados con las competencias esperadas. Parecería que el problema es local, sin embargo no lo es. En un estudio sobre el modelo por competencias mostrado por el Clayton Christensen Institute y realizado por Gallup en Estados Unidos (Weise, 2014, párr.3), las universidades aseguraban que el $96 \%$ de sus egresados cumplían con las competencias empresariales deseadas, mientras que al mismo tiempo y por su lado, los empresarios aseguraban que solo el $11 \%$ de los recién graduados recibidos por sus empresas cumplían con sus expectativas en cuanto a las competencias logradas.

Podría pensarse que lo ideal es que los empresarios participen en la hechura o definición del diseño del nuevo currículo de la educación superior, pero esta es un arma de doble filo. Se podría, quizás, realizar este levantamiento en base a las descripciones de puestos. Sin embargo, debe tenerse cuidado, pues muchas empresas privadas no trabajan basadas en las mejores prácticas del mercado. Aparte de que no son los mismos intereses los de los empresarios que buscan hacer crecer su propio capital, que los del estado, que está dado a suplir necesidades de la población en ámbitos tan vitales como la educación y la salud y, sobre todo, en ponerle fin a la pobreza. Prueba de lo delicado del caso son la gran cantidad de empresas que no cumplen con las leyes de protección medioambiental, ni con el acatamiento de las leyes laborales, entre muchos otros ejemplos palpables y conocidos por todos.
Entonces, el gobierno debe trabajar el currículo general nacional tomando en cuenta todos estos factores para posicionar los egresados en un mercado laboral, que exige profesionales con un nivel de competencias que le permitan ejercer en cualquier parte del mundo, tanto en el arte de su ciencia como en la tecnología e investigación; así como, asegurar egresados éticos y compasivos que piensen en solucionar los grandes problemas de la humanidad y propiciar la paz mundial. El levantamiento de información en este orden, aunque debe estar dirigido a suplir las necesidades nacionales, debe tener en cuenta la interdependencia entre naciones.

Asimismo, el estado podría dar facilidades a los empresarios para que puedan multiplicar sus empresas creando nuevas fuentes de trabajo para los recién egresados; establecer concesiones como el ofrecer condiciones impositivas más blandas para aquellas empresas que apoyen a las nuevas generaciones en el proceso de la inserción laboral; otorgar facilidades sobre los aranceles de importación y exportación para las compañías de manufactura; al mismo tiempo, aplicar el levantamiento de barreras a los empresarios que deseen entrar por primera vez a ciertas industrias que sean de conveniencia para la economía del país y que ingresen a sus filas jóvenes egresados. Estos pasos conllevarían la creación de alianzas estratégicas entre empresas y universidades para programas de pasantía. En fin, la creación de una estructura que concite los intereses económicos de la nación, la estabilidad del Sistema de Educación Superior; y sobre todo del Ministerio del Trabajo, con una captación de la mano de obra compuesta por la generación del nuevo milenio.

Estamos conscientes de que el gobierno y las universidades trabajan en el aseguramiento de la calidad a través de la mejoría en los insumos, procesos, productos y controles, pero aún no 
alcanzamos los estándares internacionales de países desarrollados. Y es que se trata de un proceso escalonado que acaba de ser modificado en todas sus partes. Este nuevo sistema de aprendizaje por competencias es algo de reciente implantación que está por dar sus frutos y manifestar su efectividad a largo plazo, en todos los ámbitos y zonas horarias.

En este orden de ideas, las acreditadoras juegan un papel vital ya que son los encargados de medir la calidad de lo que se hace en las universidades. Esta es una manera de certificar el cumplimiento del proyecto educacional y de asegurar la existencia y aplicación de mecanismos eficaces de autorregulación y aseguramiento de la calidad. Y es que lo que no se mide, no se cumple. Los indicadores de gestión, tanto académicos como administrativos, son de vital importancia porque aseguran el cumplimiento.

Como podemos ver, la educación del siglo XXI resulta sumamente compleja, y a esto se incorpora la época de la nanotecnología o la ciencia de lo pequeño. Por este camino van las tendencias industriales y por ahí ira la educación en cuanto a técnicas multidisciplinares que se utilizan para manipular la materia a escala de átomos y moléculas. Ya está activa su aplicación en diferentes campos, entre los que se destacan los materiales, la electrónica, la biomedicina y la energía. La influencia en la economía a nivel global es de tal dimensión que, según los expertos del Future Trend Forum, se espera que dentro de los próximos 10 años, por los productos fabricados con esta nueva tecnología, ingresen cerca de 2,5 billones de dólares en todo el mundo (Universia, 2016, 3). Y así, el mundo trabaja con la nanotecnología. Y de repente, tenemos a universidades como la de Zaragoza y su "Departamento de Física de la Materia Condensada" impartiendo Seminarios sobre Nanotecnología para estudiar el origen de la vida en la Tierra; a tal punto que términos como el nanocosmos se hacen usuales (Unizar, 2017, párr. 1)

Ya no es posible definir ni explicar las ciencias o las asignaturas desde un lugar de aislamiento, porque se conoce que no existe nada con existencia propia, nada es independiente; todo es interdependiente, interconectado. Esto hace que sea difícil para el estudiante penetrar en el conocimiento de las ciencias divididas por partes porque dificulta el conocimiento del todo y así surgen los currículos integrados. El mundo científico-tecnológico y humanístico ha reconocido que el cambio es constante: propio de los avances que el hombre genera y de nuevas leyes que se hacen evidentes cuando surgen nuevos medios para ver una realidad que antes formaba parte de los mundos ocultos. Pero hoy con los nuevos instrumentos tecnológicos; se hacen visibles y compresibles y de una utilidad increíble.

Asimismo, el conocimiento es tan cambiante como la mente cognoscitiva que lo genera. El todo está formado por sistemas y subsistemas que se interrelacionan, y que exhiben propiedades y comportamientos que a simple vista no se hacen evidentes. Un sistema complejo, al estudiarlo a profundidad, puede denotar particularidades inimaginables que surgen de la observación de un todo no predecible. Al entrelazarse, los sistemas muestran características que se producen con la acción dinámica; sin embargo, no pierden las características primarias.

La educación de hoy debe ser científica, tecnológica y humanística, pero a pesar del cambio constante, debe estar negada a la inhumanidad sin límites que prevalece. Anhelamos una educación centrada en el bien, la ética y la preservación de la naturaleza, donde se entienda que observar es observar la naturaleza, es observar todas las condiciones en el tiempo 
y el espacio. Y como bien dice el Shobogenzo: Es observar el vacío porque la naturaleza es vacía y el vacío es la naturaleza, porque el ser humano que busca encontrar la naturaleza lo logrará en el tiempo, porque el tiempo siempre llega y la naturaleza siempre está (Dogen, traducción: Bielefeldt 2010, párr. 10).

La realidad no puede entenderse sino como una multiplicidad de relaciones que la conforman, y por lo cual, todo se encuentra interconectado y cualquier deseo de separación se convierte en pura ficción. Hay una nueva relación entre el todo y las partes porque el mundo simple que teníamos en la mente como realidad conceptual, no es real; era una percepción tan errada como el que la tierra fuera plana. La realidad se construye desde el hecho mismo, desde lo vivido, conocido, desde el análisis crítico del todo y sus partes, desde la apertura a todas las posibilidades, desde el vacío que lo contiene todo, desde el todo como la plenitud del vacío.

La complejidad se aborda con mayor facilidad desde el social constructivismo; la filosofía y lo holístico. Desde una mente abierta a todas las posibilidades; la formación de mundos y esferas; la dialéctica de las formas y la ausencia de formas; el conocimiento de lo incognoscible y desde la educación del siglo XXI porque muestra lo pequeño como dueño y creador de lo grande. Partículas de luz, fotones rigiendo el mundo de la incertidumbre...

Hoy la cantidad de información en la Internet es tal, que el profesor debe enseñarle a sus estudiantes dónde buscar, cómo discriminar lo importante de la basura sin sentido, para determinar qué información o conocimiento es fiable para ser utilizado. Enseñar esto le permite al estudiante independencia en su autoaprendizaje a lo largo de la vida, porque su vida no será suficientemente larga para conocer todo lo que hay disponible para ser conocido; él deberá aprender cómo reconocer lo significativo y transcendente. La gran cantidad de información disponible lo abrumará y es ahí donde se hace importante lo que el gran pacifista Hans Kung (1990, 29) llama el "saber orientativo" y no el saber informativo. Todo conocimiento debe ser acompañado de principios y valores que propicien su uso adecuado.

Y así, hablamos de la necesidad de una ética para el conjunto de la humanidad, para los políticos y el mundo económico y financiero. De lo contrario, todas las buenas intenciones de las universidades de un mundo mejor caerán en el vacío. De la misma manera, el profesor Klaus Schwab $(2017,14)$, presidente del Word Economic Forum de Davos insiste en que:

Más que nunca, necesitaremos un liderazgo responsable y que responda para abordar nuestros desafíos colectivos y para restaurar la confianza de las personas en las instituciones y en los demás ciudadanos. No nos faltan los medios para hacer del mundo un lugar mejor. Pero para hacerlo, debemos mirar más allá de nuestros propios intereses y atender los intereses de nuestra sociedad global.

(Schwab, 2017, 14)

De igual importancia resulta el hecho de que la Educación enfrenta la conjunción de la tecnología de la comunicación del Internet y las energías renovables en el siglo XXI. Se trata de la llamada Tercera Revolución Industrial o revolución verde. Este nuevo modelo de trabajo deberá responder a las influencias sociales, tecnológicas y económicas de esta época en que nos ha tocado vivir. ¿Cómo estamos respondiendo las universidades a los efectos de esta revolución? ¿Cómo se preparan sus programas académicos para integrarla? ¿A quiénes se contrata como docentes? ¿Quiénes y dónde están los expertos? ¿Deben universidades de países en desarrollo como el nuestro participar de este tipo de reto? 
Esta nueva educación del siglo XXI debe $\mathrm{y}$ tiene que ser, si es que queremos conservar nuestro planeta y bienestar: holística, pluralista, participativa, significativa y social como respuesta al nuevo paradigma de la complejidad. Una educación realizada por las instituciones de educación superior para bienestar de todos los seres vivos y para la preservación del planeta. Una educación basada en una pedagogía que propicie una voluntad ética global que rija el accionar del profesional y de las naciones para una convivencia pacífica basada en el respeto y en el deseo de una democracia justa y duradera. Una educación que tome en cuenta a las futuras generaciones con propósitos claros, limpios, justos y necesarios para el bienestar del planeta.

\section{Referencias:}

Arnau, J. (2011). La teoría del conocimiento en Nāgārjuna. Revista Internacional de Filosofía, no 54, 2011, 125-136 ISSN: 1130-0507.

Dogen, E. (2010). Treasury of the eye of the true Dharma. [El Tesoro del ojo de la sabiduría del verdadero Dharma] Shobogenzo Bussho. Translated by Carl Bielefeldt, Soto Zen Project. Book 3. Stanford: Stanford University, 10. (Obra original publicada en el siglo XIII).

Kung, H. (1990). Proyecto de una Ética Mundial. Madrid. Trotta, p. 29.

Kung, H. (1999). En los límites de la realidad: el vacío Mundo Cientifico- La Recherche, núm. 202 (junio 1999), pp. 41-45.
Schegel, F. (1991). O dialeto dos fragmentos. Tradução de Márcio Suzuki. São Paulo: Iluminuras.

Schwab, K. (2017). Cinco prioridades de liderazgo para el 2017. En K. Schwab (Presidencia), World Economic Forum. Congreso llevado a cabo en Genova, Suiza. Recuperado de URL: https://www.weforum. org/es/agenda/2017/01/cincoprioridades-de-liderazgo-para-2017

Taeli, F. (2012). El nuevo paradigma de la complejidad y la educación: una mirada histórica. Polis [En línea], 25 | 2010, Publicado el 23 abril 2012, consultado el 18 junio 2017. URL: http://polis.revues.org/400

MEPyD (2010). Estrategia Nacional de Desarrollo 2010-2030. Santo Domingo. Ministerio de Economía, Planificación y Desarrollo de la República Dominicana. Recuperado de URL: www.economia.gob.do/ eWeb/Archivos/Libros/ Anteproyecto_de_Ley_de_END_ RD_2010_2030.pd f

Morin, E (1973). Le Paradigme perdu: Le noeud gordien de l'hominisation. [El paradigma perdido, el nudo gordiano de la humanización]. Paris: Seuil. p.92.

Morin, E. (2003). Introducción al pensamiento complejo, Gedisa, España, pp. 341342.

Morin, E. (2010). Pensar la complejidad: Crisis y metamorfosis. Valencia. Universidad de Valencia. Colección Honoris Causa. Artes Gráficas Soler, p.132. 
OCDE (2008). Informe sobre las Politicas

Nacionales de Educación:

República Dominicana, Paris:

OECD Publishing, pp. P.16

OECD (2014). Factbook 2014: Economic, Enviromental and Social Statistics. [Libro de hechos: Económicos, medioambientales y estadísticas sociales]. OECD

Publishing p.198: http://dx.doi. org/10.1787/factbook-2014-en

Universia (2006) Nanotecnología, la revoluciónindustrial del Siglo XXI. Recuperado de URL: http://noticias.universia. es/ciencia-nn-tt/nocia/2006/05/18/598810/nanotecnologia-revolucion- industrial-siglo-xxi.html
Unizar (2017). Nanotecnología para estudiar el origen de la vida en la Tierra. Seminarios 2017 del Departamento de Física de la Materia Condensa da. Universidad de Zaragoza.

Recuperado de URL: https://ciencias. unizar.es/actividad/semina rios-2017-del-departamento-de-fisica-de-la-materia-condensada-nanotecnologia-para

Weise, M. (2014). Higher Education:

Online Competency-based

Learning. Disruptive innovation. [Educación superior: Aprendizaje basado en competencias, en línea. Innovación disruptiva]. Clayton Christensen Institute. Recuperado de URL: https:// youtu.be/hOJM6Hv1I8g

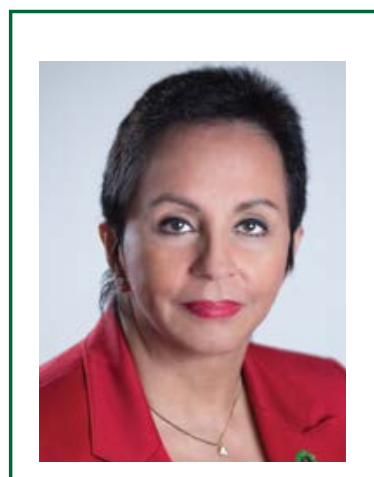

\section{Ofelia Berrido}

Narradora interiorista, ensayista, poeta y gestora cultural dominicana. Actualmente escribe ensayos para la Revista Cultural Areíto del periódico "HOY", así como para el diario digital español Latin Press. Es Directora del Departamento de Tecnología Educativa y de la Unidad de Evaluación y Desarrollo Docente UNPHU, así como de la revista AULA. Formadora de formadores del Modelo Educativo UNPHU. Ha sido docente del área de postgrado de la UNPHU y de UNIBE y del área de Lengua y Literatura de esta última. Es Miembro Correspondiente de la Academia Dominicana de la Lengua; del Ateneo Insular y del Grupo Mester de Narradores de la Academia. Es además Miembro de "Society of Human Resources Management". Berrido es graduada de Doctora en Medicina por la Universidad Autónoma de Santo Domingo con una especialidad en Ginecología y Obstetricia de la Escuela de Residencias Médicas de la MNSA-UASD, y una subespecialidad en Oncología Ginecológica de la mismas instituciones; ha realizado cursos en la Universidad de Río Piedras, Puerto Rico y en el Hospital John Hopkins, Baltimore. Cuenta; además, con una Maestría en Administración de Negocios por la prestigiosa "Braniff School of Management of the University of Dallas" y varios Diplomados, entre ellos el Diplomado de Certificación Docente UNPHU. Realizó, asimismo, diversos cursos de Recursos Humanos en Abbott Hospitals, Chicago. Es asesora de "Planificación Estratégica y Recursos Humanos y ha trabajado en ambas disciplinas tanto local como internacionalmente para importantes empresas e instituciones locales e internacionales como lo son Abbott Hospitals, GTE y Verizon Headquarters. 